\title{
What are the arterial oxygen saturation discrepancies in some pediatric diseases and the risk factors for oxygen desaturation?
}

\author{
Heba Safar \\ Department of Pediatrics, Faculty of Medicine, Fayoum University. \\ Telephone: 00201222712983 \& 00201091881160 \\ E-mail address: safarheba@yahoo.com , hebasafar97@gmail.com \\ Postal Address: Fayoum Egypt.63511
}

\begin{abstract}
:
Aim of work: Current study aims to establish the reference range profiles for oxygen saturation by pulse oximetry among three groups of some pediatric diseases. The study pediatric population selection based upon association between their underlying diseases and occurrence of oxygen desaturation. They are classified into group of cyanotic congenital heart disease, group of acyanotic congenital heart disease and third group of $\beta$-thalassemia major patients.

Methods: Descriptive Cross sectional study was conducted in the department of Pediatrics, Faculty of Medicine, Al-Fayoum University hospital from September 2017 to June 2018. Study group 1,2 consisted of 68 children from 2month-8 years had congenital heart diseases (35 cyanotic represents group 1) \& (33 acyanotic represents group 2 ). 40 patients from $3.5-12$ years with $\beta$ -

thalassemia major who receive regular blood transfusion and iron chelation represents group 3. All study groups' arterial oxygen saturation values were measured using pulse oximetry; $\mathrm{SpO} 2$ data were then collected and presented as reference range of arterial oxygen saturation for each group. Investigation of possible risk factors for desaturation for each group was done.

Results and conclusion: reference range $72 \%-80.5 \%$ for cyanotic congenital heart disease study group, $89.25 \%$ - $92.9 \%$ for acyanotic congenital heart disease study group and $96.2 \%-97.4 \%$ for $\beta$-thalassemia major study group were estimated. Stages of heart failure which is presented as NYHA (New York Heart Association) Classification is found to be one of the risk factors for oxygen desaturation in acyanotic congenital heart disease group.
\end{abstract}

\section{KEYWORDS:Arterial oxygen}

saturation, children, reference range

\section{INTRODUCTION:}

Pulse oximetry is considered an inexpensive, noninvasive and generally reliable method to assess oxygenation. It is suggested to be the fifth vital sign. [1]

Despite its importance and reliability the exact interpretation of pulse oximetry readings in healthy children has not yet been clearly established. Normal pediatric oxygen saturation values, reference range show discrepancies between references. For example, normal arterial oxygen saturation values have been reported as 
94-99\% [2], 95-97\% [3] by two

different references.

In practice, however, the $\mathrm{SpO} 2$ range of $92-100 \%$ is generally accepted for most health care givers. Some experts have found that a $\mathrm{SpO} 2$ level of at least $90 \%$ will prevent hypoxic tissue injury and achieve patient safety. [4]

The pulse oximetry screening programs is beneficial to pick up desaturated infants and children through their SpO2.These children may be undiagnosed and experience desaturation due to certain pediatric diseases that impair normal arterial oxygen saturation.

Also, $\mathrm{SpO} 2$ reference range can be used as a guide to compare and assess $\mathrm{SpO} 2$ profiles of infants and children on home oxygen therapy.

However, measuring arterial oxygen saturation by pulse oximetry is unreliable in cases of hypothermia, hypovolaemia, shock, severe hyperoxia ( $\mathrm{PaO} 2$ more than 100) or severe hypoxia ( $\mathrm{PaO} 2$ less than 70$)$ and in other conditions known to interfere with oxygen saturation, including smoke inhalation with carbon monoxide poisoning, the use of intravenous dyes, nitroglycerin, nail polish, direct sunlight.[5]

In these cases, an ear lobe pulse oximeter device or arterial blood gases

\section{METHODS:}

Descriptive cross sectional study was conducted in the department of Pediatrics, Faculty of Medicine, AlFayoum University hospital from September 2017 to June 2018.

\section{Inclusion criteria:}

Study group 1,2 consisted of 68 children from 2month- 8 years had congenital heart diseases ( 35 cyanotic represents group 1) \& (33 acyanotic represents group 2 ). 40 patients from 3.5 -12 years with $\beta$-thalassemia major who receive would give a more precise oxygen saturation level. However, arterial blood gases are usually only done in intensive care units or emergency room. [6]

Many studies were done to define the exact reference range of arterial oxygen saturation in healthy children but small number of studies was done to construct reference range of arterial oxygen saturation in children who experience desaturation due to different underlying diseases. Knowing more information about arterial oxygen saturation of some pediatric diseases help in their early diagnosis during pulse oximetry screening programs and enhance better management by oxygen therapy or interventions that eliminate the etiology for desaturation as for example, surgical interventions for congenital heart diseases.

Current study aims to establish the reference range profiles for oxygen saturation by pulse oximetry among three groups of some pediatric diseases. The study pediatric population selection based upon association between their underlying diseases and occurrence of desaturation. Subsequently, they are classified into group of cyanotic congenital heart disease, group of acyanotic congenital heart disease and third group of $\beta$-thalassemia major patients.

regular blood transfusion and iron chelation represent study group 3.All three study groups were randomly selected from patients who attended inpatient wards of Fayoum University Hospital.

\section{Exclusion criteria:}

Patients with associated extracardiac malformations or genetic disorders, children with isolated cardiac arrhythmias, cardiomyopathy, acquired heart disease, isolated dextrocardia, 
cardiac tumors and mitral valve leaflet prolapse were excluded from the study. Other causes of desaturation, and critically ill children were excluded.

Demographic characters and anthropometric measures in different study groups were done.

All cases are subjected to detailed history taking, full clinical examination with focus on pallor, cyanosis, respiratory rate, heart rate, 1hepatomegaly and other cardiac symptoms, chest $\mathrm{x}$-ray, ECG. The Mmode, two-dimensional, and Doppler echocardiography by using Vivid 3 with $5 \mathrm{MHz}$ transducer phase array scanner, Norway were done. Echocardiography was performed by a pediatric cardiologist to confirm diagnosis of congenital heart disease (both cyanotic and acyanotic heart disease).

For patients with $\beta$-thalassemia major each patient in the study had a clinical and hematological data file including duration of disease, cardiovascular assessment for pulse rate, apex beat, blood pressure, heart sounds and presence or absence of murmur, and body weight. Data of the blood hemoglobin, serum ferritin concentrations were all extracted.

All study groups' arterial oxygen saturation values were measured using pulse oximetry probe connected to a bedside monitor (Nihon Kohden, Japan) through pediatric $\mathrm{SpO} 2$ connection cords; SpO2data were then collected and presented as reference ranges of arterial oxygen saturations for each group.

In a study 2015, although the traditional information is that pulse oximetry values $95 \%$ or greater are normal, these data suggest that the normal oxygen saturation range should be between $97 \%$ and $100 \%$. Values of $95 \%$ and $96 \%$ should guide clinician to the presence of underlying disease. [7]
Based on these results, the normal oxygen saturation level is considered $97-100 \%$ in the present study.

Correlation of age and weight as possible risk factors for desaturation for each group was done. In addition investigation of other possible risk factors for desaturation for each group was done as follows:

For cyanotic CHD group, echocardiographic assessment for degree of pulmonary stenosis pressure gradient, if present, as one of the risk factors for oxygen desaturation in addition to structural abnormalities that causes mixing of oxygenated and deoxygenated blood.

2- For acyanotic CHD group, tachycardia, presence of heart failure, NYHA classification (New York Heart Association classification) that represent the stages of heart failure and history of recurrent chest infections were investigated as a common risk factors for oxygen desaturation.

NYHA Classification - The Stages of Heart Failure: [8]

Class I - No symptoms and no limitation in ordinary physical activity, e.g. shortness of breath when walking, climbing stairs etc.

Class II - Mild symptoms (mild shortness of breath and/or angina) and slight limitation during ordinary activity.

Class III - Marked limitation in activity due to symptoms, even during less-thanordinary activity, e.g. walking short distances (20-100 m).Comfortable only at rest.

Class IV - Severe limitations. Experiences symptoms even while at rest. Mostly bedbound patients.

3- For $\beta$-thalassemia major group, tachycardia and presence of heart failure were investigated as risk factors for desaturation. 
Difference of arterial oxygen saturation between different ages in each study group was done.

\section{ETHICAL COMMITTEE:}

The study was reviewed and approved by the Faculty of Medicine Research Ethical Committee. A waiver and verbal consent was obtained by the

\section{STATISTICAL ANALYSIS:}

Data were collected and coded to facilitate data manipulation and double entered into Microsoft Access and data analysis was performed using Statistical Package of Social Science (SPSS) software version 18 in windows 7. Simple descriptive analysis in the form of numbers and percentages for qualitative data, and arithmetic means as central tendency measurement, standard deviations as measure of dispersion for quantitative parametric data. Quantitative data included in the study was first tested for normality by OneSample Kolmogorov-Smirnov test in each study group then inferential statistic tests were selected.

\section{RESULTS:}

Demographic characters in different study groups illustrate that there is a higher mean of age among patients with $\beta$ - thalassemia major (8.1 \pm 3.1$)$ with and low mean among both cyanotic, and acyanotic congenital heart disease groups. Also as regards sex distribution with higher percentage of females were noted among acyanotic congenital heart disease group, and $\beta$ thalassemia major patients, but higher percentage of males among cyanotic congenital heart diseasegroups. author after proper orientation of the care givers regarding the objectives of the study.
- For quantitative parametric data :

One way ANOVA test in comparing more than two independent groups of quantitative data.

\section{- For qualitative data}

- Chi square test to compare two of more than two qualitative groups.

- Bivariate Pearson correlation test to test association between variables

- Multiple linear regressions to test association between quantitative dependent and in-dependent variables and detection of risk factors.

- The P-value $\leq \mathbf{0 . 0 5}$ was considered the cut-off value for significance.

Anthropometric measures in different study groups illustrate that the higher mean of weight(23.5 \pm 8.3$)$ and weight percentiles $\left(25 \mathrm{th} \pm 25^{\text {th }}\right)$ among patients with $\beta$-thalassemia major and low mean among both cyanotic, and acyanotic congenital heart disease groups. As regards levels of weight percentiles between three study groups higher percentage of all groups were 3 rd percentile. More than half of children among acyanotic CHD group were normal in weight but both cyanotic CHD group and $\beta$-thalassemia major group show low weight. 
Table (1) illustrates the Mean, Median and Reference range (95\% Confidence Interval) of oxygen saturation among patients with reference range $\mathbf{7 2 \%} \mathbf{- 8 0 . 5 \%}$ for cyanotic congenital heart disease study group, $\mathbf{8 9 . 2 5 \%} \mathbf{- 9 2 . 9 \%}$ for acyanotic congenital heart disease study group and $\mathbf{9 6 . 2 \%} \mathbf{- 9 7 . 4 \%}$ for $\beta$-thalassemia major study group.

Table (2) illustrates that different clinical finding among cyanotic congenital heart disease group with $97.1 \%$ had desaturation of oxygen with mean oxygen saturation level of (76.3 \pm 12.4$)$ and mean pressure gradient of pulmonary stenosis of $(55.3 \pm 29.2)$

Table (3)illustrates that different clinical finding among acyanotic congenital heart disease group with $72.7 \%$ had recurrent chest infection, and had heart failure, 97\% show oxygen desaturation with mean oxygen saturation level of (91.1 $\pm 5.5)$ and mean heart rate of (140 \pm 15 ).

Table (4) illustrates that different clinical finding among $\beta$ thalassemia group with $45 \%$ show heart failure and $52.5 \% \%$ had desaturation of oxygen with mean oxygen saturation level of $(96.8 \pm 1.9)$ and mean heart rate of $(96 \pm 15)$

Table (5) and figure $(1,2)$ illustrates that there is statistically significant positive correlation with p-value $<0.05$ between oxygen saturation level with age and weight in the whole study group; which

\section{DISCUSSION:} oxygen saturation level changes according to person's health status. Thus, it is important to understand both normal reference range for healthy subjects and underlying indicated increase in age and weight of child will associated with increase in oxygen saturation level. On the other hand there is no statistically significant correlation with $\mathrm{p}$-value $>0.05$ between oxygen saturation level with age and weight in each of the three study groups.

Table (6) illustrates the multivariate linear regression model analysis that was conducted to explore the explanatory power of different risk factors in prediction of oxygen desaturation; it illustrates that there was statistical significance predictors with $\mathrm{p}$-value 0.05 to NYHA classification among acyanotic congenital heart disease group; but no effect of other factors (age, weight, heart rate, heart failure, or recurrent chest infection), also there is no statistical significance predictors with $p$-value $>0.05$ to all variables in detection of oxygen desaturation among cyanotic congenital heart disease or $\beta$ thalassemia major groups.

Table (7) illustrates that there is statistical significant difference in oxygen saturation among different age groups with statistical significant low mean of oxygen saturation among patients aged between 1-3 years among acyanotic congenital heart disease group with p-value 0.01 , but no statistical significant difference in oxygen saturation among different age group in both cyanotic congenital heart disease and $\beta$-thalassemia groups.

pathology associated with certain diseases to interpret oxygen saturation levels and discrepancies in these levels.

Current study investigated three pediatric diseases known to have arterial oxygen desaturation which is congenital 
heart diseases, both, cyanotic and acyanotic, and $\beta$-thalassemia major group.

People who are, obese and/or have conditions such as lung and cardiovascular diseases, emphysema, chronic obstructive pulmonary disease, congenital heart disease and sleep apnea show lower oxygen saturation levels. [9]

For cyanotic congenital heart disease, the current study establishes a reference range of $\mathbf{7 2 \%} \mathbf{- 8 0 . 5 \%}$.

Mixing of deoxygenated blood with oxygenated blood due to structural abnormalities in cyanotic congenital heart disease, resulting in cyanosis, can occur at the level of the systemic veins, atria, ventricles, great vessels, and at distal pulmonary vascular bed. [10]

In cyanosis with decreased pulmonary blood flow: The most common causes are right-sided obstructive lesions that result in inadequate blood flow to the lungs. Assisted ventilation and increased environmental oxygen are often necessary. For these infants, oxygen saturations in the $75-85 \%$ range are adequate. [11]

Current study evaluated pulmonary stenosis pressure gradient, as one of the commonest right-sided obstructive lesions associated with cyanotic congenital heart diseases, for being risk factor for oxygen desaturation. No effect of pulmonary stenosis pressure gradient was found.

For acyanotic congenital heart disease, the current study establishes a reference range of $\mathbf{8 9 . 2 \% - 9 2 . 9 \%}$.

Acute lower respiratory tract infection is the leading cause of death in children below 5 years of age in developing countries and hypoxaemia is the major fatal complication. Acyanotic congenital heart diseases present with hypoxaemia due to common complications as recurrent lower respiratory tract infections and heart failure.
Similarly, in a study done 2005; they found that, although oxygen saturation of $95 \%$ and $96 \%$ are adequate. These values are associated with higher APC/RI (Airway, Pulmonary and cardiac problems/Respiratory infections) percentages and thus should be considered potentially abnormal. An oxygen saturation of $97 \%$ is on the border of normal. Normal oxygen saturation can occur with APC/RI conditions, but oxygen saturation less than $97 \%$ is associated with a higher risk of an APC/R1 condition. [12]

Current study found that heart failure which is presented as NYHA (New York Heart Association) Classification which represents stages of heart failure, as one of the risk factors for oxygen desaturation in acyanotic congenital heart disease group.

For $\beta$-thalassemia major group, the current study establishes a reference range of $96.2 \%-97.4 \%$.

Oxygen saturation may remain normal (e.g., 97\% and higher) for people with anemia. However, this may not indicate adequate oxygenation because there is less hemoglobin to carry an adequate supply of oxygen for people who have anemia. The inadequate supply of oxygen may be more prominent during activity for people with anemia.[9]

This might explain results of current study as nearly half of $\beta$-thalassemia major group presented with anemic heart failure and consequently, nearly half of them show oxygen desaturation levels.

Special care and oxygen therapy for patients aged between 1-3 years among acyanotic congenital heart disease group will help them as current study showed statistical significant low mean of oxygen saturation among this age groups. 
The presented reference values in the three study groups may be used for clinical decision making. Therefore, defining hypoxaemia by oxygen saturation reference range will help to

\section{STUDY LIMITATIONS:}

Larger sample size is needed to confirm reference range values for oxygen saturation. Also, further studies are needed

\section{CONCLUSION:}

Reference range $72 \%-80.5 \%$ for cyanotic congenital heart disease study group, $89.25 \%$ - $92.9 \%$ for acyanotic congenital heart disease study group, and $96.2 \%$ - $97.4 \%$ for $\beta$-thalassemia major study group were estimated. Heart failure which is presented as NYHA (New York Heart Association) Classification is found to

\section{REFERENCES:}

[1] Safar HH, El-dash HH. Pulse Oximetry. Could Wrist and Ankle Be Alternative Placement Sites?. Clin Pediatr. 2015; Vol. 54(14): 1375-1379.

DOI: $10.1177 / 0009922815584217$

[2] Baker WE. Lanoix, R. Field. DL. Hedges. JR. Noninvasive Assessment and Support of Ovygenation and Ventilation. In: Roberto, cit. Clinical Procedures in Emergency Medicine. 3rd ed. Philadelphia. PA W. B Saunders Company. 1998: 82-107. [3] Sondheimer, PM, Boucek, MM, Ivy, D, Loris. A. Schaffer MS. Wolfe, RR. Cardiovascular Diseases, In: Hay. Jr. WW, Hayward AR. Levis MJ. SondheimerJM, eds, Current Pediatric Diagnosis and treatment.16th ed. New York, NY: Lange Medical Books: 2003:544-613.

[4] Beasley R,Chien J, Douglas J. Thoracic Society of Australia and New Zealand oxygen

guidelines for acute oxygen use in adults:

'Swimming between the flags. Respirology. 2015; 20: 1182-1191.

[5] Rufo PA, Loughlin J, Johnson KB. The Harriet Lane Handbook, 13th edn. St Louis: Mosby Year Book, 1993; 340-1. establish definite limits for earlier diagnosis, supplementing oxygen in various age groups, and minimize cost of oxygen consuming without urgent need.

to include all pediatric diseases that result in oxygen desaturation.

be one of the risk factors for oxygen desaturation in acyanotic congenital heart disease group. Subsequently, considering these values help to categorize and predict some diseases that causes desaturation and offer early diagnosis and management during pulse oximetry screening programs and ensure proper oxygen therapy.

[6] Mengelkoch LJ, Martin D, Lawler J. A review of the principles of pulse oximetry and accuracy of pulse oximeter estimates during exercise. Phys Ther. 1994;74:40-49.

[7] Elder JW1, Baraff SB, Gaschler WN, Baraff LJ. Pulse Oxygen Saturation Values in a Healthy School-Aged Population. Pediatr Emerg Care. 2015 Sep;31(9):645-7.

doi: 10.1097/PEC.0000000000000331.

[8] Dolgin M, Committee NYHAC. In: Dolgin M, editor. Nomenclature and criteria for diagnosis of diseases of the heart and great vessels. 9th ed. Boston: Little, Brown; 1994.

[9] Lapum JL, Verkuyl M, Garcia W. What are Normal Oxygen Saturation Levels?In: Vital Sign Measurement Across the Lifespan - 1st Canadian edition. Ecampusontario Toronto. 2015;107-108.

[10] Jaganmohan A Tharakan. Admixture lesions in congenital cyanotic heart disease Ann Pediatr Cardiol. 2011 Jan-Jun; 4(1): 5359.

doi: 10.4103/0974-2069.79625 
[11] Christopher W. Mastropietro, Susan P. Tourner, Ashok P. Sarnaik. Emergency Presentation of Congenital Heart Disease In Children. Pediatr Emerg Med. May 2008 ; 5 ( 5):1-32.
[12] Mau MK, Yamasato KS, Yamamoto LG.Normal Oxygen Saturation Values in Pediatric Patients.Hawai Med J.2005;42-45.

Table (1): Reference range for arterial oxygen saturation levels in different study groups.

\begin{tabular}{|l|c|c|c|}
\hline \multirow{2}{*}{\multicolumn{1}{|c|}{ Groups }} & \multicolumn{3}{|c|}{ Oxygen saturation } \\
\cline { 2 - 4 } & Mean \pm SD & Median & $\begin{array}{c}\text { Reference range } \\
(95 \% \text { CI })\end{array}$ \\
\hline Cyanotic CHD & $76.3 \pm 12.4$ & 75 & $\mathbf{7 2}-\mathbf{8 0 . 5}$ \\
\hline Acyanotic CHD & $91.1 \pm 5.5$ & 92 & $\mathbf{8 9 . 2}-\mathbf{9 2 . 9}$ \\
\hline $\begin{array}{l}\boldsymbol{\beta} \text {-Thalassemia } \\
\text { Major }\end{array}$ & $96.8 \pm 1.9$ & 97 & $\mathbf{9 6 . 2}-\mathbf{9 7 . 4}$ \\
\hline
\end{tabular}

Table (2): Clinical findings among cyanotic congenital heart diseases group.

\begin{tabular}{|l|c|c|}
\hline \multirow{2}{*}{ Variables } & \multicolumn{2}{c|}{ Frequency } \\
\cline { 2 - 3 } Level of oxygen saturation & No. & \% \\
\hline Normal & 1 & $2.9 \%$ \\
\hline Desaturation & 34 & $97.1 \%$ \\
\hline Other findings & Mean & SD \\
\hline Level of oxygen saturation & 76.3 & 12.4 \\
\hline Pulmonary stenosis & 55.3 & 29.2 \\
\hline
\end{tabular}

Table (3): Clinical findings among acyanotic congenital heart diseases group.

\begin{tabular}{|c|c|c|}
\hline \multirow{2}{*}{ Variables } & \multicolumn{2}{|c|}{ Frequency } \\
\cline { 2 - 3 } NYHA classification & No. & $\%$ \\
\hline Class I & 11 & $33.3 \%$ \\
\hline Class II & 8 & $24.2 \%$ \\
\hline Class III & 9 & $27.3 \%$ \\
\hline
\end{tabular}


ISSN: 2536-9482 (Online) Fayoum University Medical Journal $\quad$ Safar et al., 2019,2(1), 59-70

\begin{tabular}{|c|c|c|}
\hline \multicolumn{2}{|l|}{5} & $15.2 \%$ \\
\hline Recurrent chest infection & 9 & $27.3 \%$ \\
\hline No & 24 & $72.7 \%$ \\
\hline Yes & 9 & $27.3 \%$ \\
\hline Heart failure & 24 & $72.7 \%$ \\
\hline No & \multicolumn{2}{|l}{} \\
\hline Yes & 1 & $3 \%$ \\
\hline Level of oxygen saturation & 32 & $97 \%$ \\
\hline Normal & Mean & SD \\
\hline Desaturation & 91.1 & 5.5 \\
\hline Other findings & 140 & 15 \\
\hline Level of oxygen saturation &
\end{tabular}

Table (4): Clinical findings among $\beta$-thalassemia major group.

\begin{tabular}{|c|c|c|}
\hline \multirow{2}{*}{ Variables } & \multicolumn{2}{|c|}{ Frequency } \\
\cline { 2 - 3 } Heart failure & No. & $\%$ \\
\hline No & 22 & $55 \%$ \\
\hline Yes & 18 & $45 \%$ \\
\hline Level of oxygen saturation & \multicolumn{2}{|c|}{} \\
\hline Normal & 19 & $47.5 \%$ \\
\hline Desaturation & 21 & $52.5 \%$ \\
\hline Other findings & Mean & SD \\
\hline Level of oxygen saturation & 96.8 & 1.9 \\
\hline Heart rate & 96 & 15 \\
\hline
\end{tabular}


Table (5): Correlation between oxygen saturation levels with age and weight in whole study group, and in each group.

\begin{tabular}{|c|c|c|}
\hline \multirow{2}{*}{ Variables } & \multicolumn{2}{c|}{ Oxygen saturation } \\
\cline { 2 - 3 } & r & p-value \\
\hline Whole study group & $\mathbf{0 . 4 5}$ & $\mathbf{0 . 0 0 1}$ * \\
\hline Age (years) & $\mathbf{0 . 4 4}$ & $\mathbf{0 . 0 0 1}$ * \\
\hline Weight (kg) & 0.09 & 0.6 \\
\hline Cyanotic CHD & 0.009 & 0.9 \\
\hline Age (years) & & \\
\hline Weight (kg) & 0.04 & 0.8 \\
\hline Acyanotic CHD & 0.09 & 0.6 \\
\hline Age (years) & & \\
\hline Weight (kg) & 0.03 & 0.8 \\
\hline B-thalassemia major & -0.07 & 0.6 \\
\hline Age (years) & & \\
\hline Weight (kg) & & \\
\hline
\end{tabular}

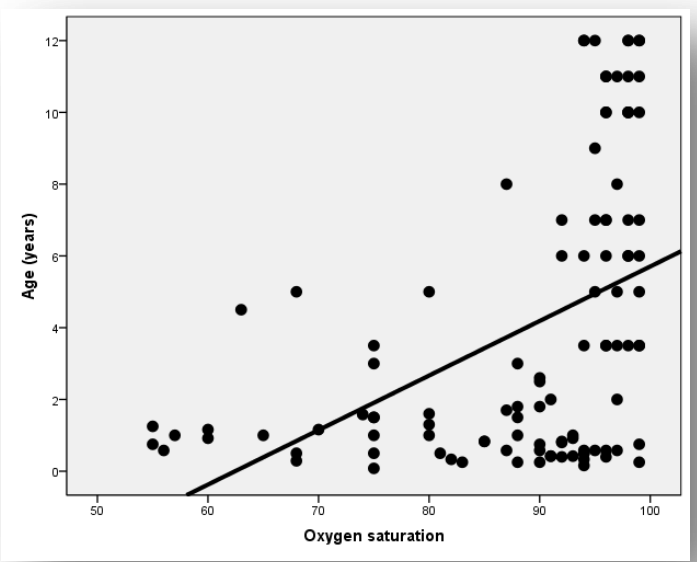

Figure(1):Correlation between of oxygen saturation with age in whole study group. 


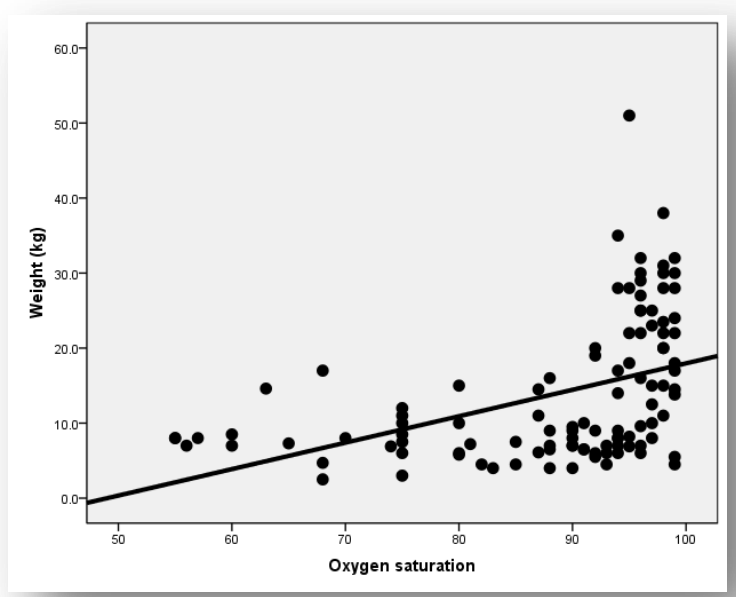

Figure(2):Correlation between of oxygen saturation with weight in whole study group.

Table (6): Linear regression analysis to determine the risk factors of oxygen desaturation in each study group.

\begin{tabular}{|l|l|l|l|}
\hline \multicolumn{1}{|c}{ Bariables } & \multicolumn{1}{c}{ SE } & Sig \\
\hline Cyanotic CHD group & 1.9 & 2.6 & 0.5 \\
\hline Age (years) & -1.1 & 1.4 & 0.4 \\
\hline Weight (kg) & 0.006 & 0.09 & 0.9 \\
\hline $\begin{array}{l}\text { Pulmonary stenosis pressure } \\
\text { gradient }\end{array}$ & & \\
\hline $\begin{array}{l}\text { Acyanotic CHD group } \\
\text { Age (years) }\end{array}$ & -0.93 & 1.9 & 0.6 \\
\hline Weight (kg) & 0.43 & 0.64 & 0.5 \\
\hline NYHA classification & -2.2 & 1.1 & $\mathbf{0 . 0 5}$ \\
\hline Heart rate & 0.06 & 0.1 & 0.6 \\
\hline Recurrent chest infection & -1.1 & 2.3 & 0.6 \\
\hline Heart failure & -0.39 & 2.8 & 0.9 \\
\hline $\boldsymbol{\beta}$-Thalassemia major group & 0.21 & 02 & 0.3 \\
\hline Age (years) & & \\
\hline
\end{tabular}


ISSN: 2536-9482 (Online) Fayoum University Medical Journal $\quad$ Safar et al., 2019,2(1), 59-70

\begin{tabular}{|l|l|l|l|}
\hline Weight $(\mathrm{kg})$ & -0.09 & 0.07 & 0.2 \\
\hline Heart rate & -0.003 & 0.04 & 0.9 \\
\hline Heart failure & 0.42 & 1.1 & 0.7 \\
\hline
\end{tabular}

Table (7): Oxygen saturation level in different age group in each study group.

\begin{tabular}{|c|c|c|c|}
\hline Age groups & $\begin{array}{l}\text { cyanotic } \\
(n=35)\end{array}$ & $\begin{array}{c}\text { acyanotic } \\
(n=33)\end{array}$ & $\begin{array}{c}\boldsymbol{\beta} \text {-thalassemia } \\
(\mathrm{n}=40)\end{array}$ \\
\hline$<1$ years old & $76.6 \pm 13.9$ & $92.3 \pm 3.2$ & ----- \\
\hline 1-3 years old & $76.4 \pm 12$ & $87 \pm 7.7$ & $97 \pm 1.8$ \\
\hline 4-6 years old & $70.3 \pm 8.7$ & $95.5 \pm 0.71$ & $96.7 \pm 2.7$ \\
\hline $7-10$ years old & $87 \pm 0$ & $\begin{array}{ll}---- \\
\end{array}$ & $96.6 \pm 1.9$ \\
\hline$>10$ years old & ----- & ----- & $96.9 \pm 1.8$ \\
\hline p-value & 0.7 & $0.01 *$ & 0.9 \\
\hline
\end{tabular}

